\title{
Ecological-coenotic features of rare flora species of pine-oak stands of Volyn Polissya
}

\author{
S. O. Glinska, S. S. Shtokalo, D. V. Lyko, Ya. V. Stepaniuk, L. K. Savchuk \\ Rivne State University of the Humanities, Ukraine
}

E-mail: glynska@ex.ua

Received 13.01.2020 Accepted 13.02.2020

\begin{abstract}
Anthropogenic influence on the natural vegetation of Volyn Polissya threatens the existence of habitats of rare and endangered species of flora. Therefore, the region, unique in botanical and geographical terms, is gradually losing its specific vegetation characteristics. Having analyzed the literature data, herbarium data and materials of our own field studies in 2016-2019, we have compiled a list of rare and endangered species of pine-oak stands. In the habitat of pine-oak stands 89 rare species were found, 29 of which are listed in the Red Data Book of Ukraine, while Silene lithuanica is included in the European Red List. 3 species (Cypripedium calceolus, Trapa natans ta Caldesia parnassifolia) are included into appendices of "The Convention on International Trade in Endangered Species of Wild Fauna and Flora". 10 species are subject to protection according to the appendix of "Convention on the Conservation of European Wildlife and Natural Habitats" 56 species are regionally rare species for the flora of the Volyn region, 4 species are rare species of pine-oak stands. In our research we have analyzed the age range, density and recovery index for the species studied. The study found that for 63 rare species the dynamics of species abundance and distribution are satisfactory. The area of distribution and the number of 7 species is increasing: Allium ursinum, Galanthus nivalis, Platanthera chlorantha, Anemone sy/vestris, Campanula persicifolia, Corydalis cava, Isopyrum thalictroides In the study area 14 species grow sporadically: Juniperus communis, Potentilla alba, Digitalis grandiflora, Gymnocarpium dryoptheris, Daphne mezereum, Neottia nidus-avis, Epipactis helleborine, Scorzonera purpurea, Asparagus officinalis, Iris sibirica, Adonis vernalis, Cephalanthera damasonium, Gentiana cruciate, Gentiana pneumonanthe. Dissemination information for Caldesia parnassifolia, Succisella inflexa, Genistella sagittalis, Salix myrtilloides, Ophioglossum vulgatum is insufficient for establishing species dynamics and needs further investigation. The conservation of pine-oak stands in Volyn Polissya will help to create the conditions for the growth of rare and endangered species of flora.
\end{abstract}

Keywords: Rare species; Population; Age spectrum of the population

\section{Introduction}

Anthropogenic influence on the natural vegetation of Volyn Polissya threatens the existence of habitats of rare and endangered species of flora. Therefore, the region, unique in botanical and geographical terms, is gradually losing its specific vegetation characteristics. Information about the rare species of the flora of Volyn Polissya is given in the works of researchers, which mainly covered information about their geographical distribution (Vandas, 1986; Pachoski, 1900; Macko, 1937; Andrienko, et al., 2004, 2009). The current status of rare flora species of pine-oak stands of Volyn Polissya has not been sufficiently investigated, there are no satisfactory data on the geographical location of the localities and the state of the populations of the species, there is no general analysis of the horological patterns. The purpose of the research is to establish ecological-coenotic features of rare flora species of pine-oak stands of Volyn Polissya.

To achieve this goal, the following objectives were set:

- To compile a list of rare and endangered species of flora;

- To conduct a sozological analysis of rare species;

- To establish dynamic trends in the geographical distribution of rare flora species.

Therefore, studying the features of the current distribution of rare flora species, monitoring their status and population structure is an urgent and important task, without which there are risks of the extinction of some rare species from the natural flora of the region.

\section{Objectives and Research Methods}

Field studies of rare and endangered species were conducted from 2016 to 2019. During this time, locations known for literary and herbarium data were checked and new rare species were found. Field surveys were carried out annually using forwarding methods in the territory of the Lopatyn, Ozersk, and Zviriv Nature Conservation Departments of Kivertsi National Natural Park "Tsumanska Pushcha".

For each botanical permanent sampling area (BA) a passport was established, a geobotanical description was made, and the age spectrum of the population was analyzed. Phytocoenotic descriptions were performed by the method (Rabotnov, 1978). Population age structure and their types by age composition have been studied by methods (Uranov, 1975; Rabotnov, 1978). The analysis of 
the coenopopulations of rare species of flora was carried out on the basis of the quantitative ratio of plants of different ontogenetic states in permanent sampling areas. The study identified four age groups: juvenile (j), immature (im), virginal (v) and generative (g). For the analysis of the dynamics of self-sustaining population, the RI - renewal index was used as the ratio of the number of preregenerative specimens to the number of generative plants.

\section{Results of the research}

Information on the distribution of rare species of flora is given in the works of researchers of Volyn Polissya.

For the outskirts of the Tsuman village four rare species are listed as habitats: Betula humilis Schrank., Anemone sy/vestris L., Cimicifuga europaea Schipcz., Clematis recta L. (Vandas, 1886). By studying the flora of Polissya, the author points at seven habitats of rare species of the studied area: Cypripedium calceolus L., Platanthera bifolia (L.) Rich., Betula humilis, Anemone sylvestris, Melittis sarmatica L., Cimicifuga europaea, Clematis recta (Paczoski, 1900). In describing the vegetation cover of the projected reserve "Yulana Area" in the vicinity of Lutsk are given Gladiolus imbricatus L., Lilium martagon L., Anemone sylvestris, Campanula cervicaria L., Clematis recta (Macko, 1937). 21 species of vascular plants from the Red Data Book of Ukraine were found within the Kivertsi National Nature Park "Tsumanska Pushcha" (Caldesia parnassifolia (L.) Parl., Allium ursinum L., Galanthus nivalis L., Gladiolus imbricatus, Carex umbrosa Host., Lilium martagon, Cephalanthera longifolia (L.) Fritsch., Cypripedium calceolus, Dactylorhiza incarnata (L.) Soo., Dactylorhiza fuchsii (Druce) Soo., Epipactis helleborine (L.) Crantz., Epipactis atrorubens (Hoffm. ex Bernh.) Besser., Neottia nidus-avis (L.) Rich., Platanthera bifolia, Platanthera chlorantha (Cust.) Rchb., Betula humilis Schrank., Genistella sagittalis (L.) Gams., Salix myrtilloides L., Iris sibirica L., Succisella inflexa (Kluk) G. Beck., Silene lithuanica Zapal.); 9 regionally rare species (Astrantia major L., Polypodium vulgare L., Ophioglossum vulgatum L., Gymnocarpium dryoptheris (L.) Newm., Cimicifuga europaea, Nimphaea candida C. Presl., Campanula persicifolia L., Melittis sarmatica, Trollius europaeus L.); 3 rare species (Aquilegia vulgaris L., Nimphaea alba L., Lonicera xylosteum L.) (Andrienko, et al., 2004). During the study of flora of the Volyn region, the habitat of rare species for Kivertsi National Nature Park "Tsumanska Pushcha" is given for: Lycopodium annotinum L., Allium ursinum, Galanthus nivalis, Carex umbrosa, Lilium martagon, Cephalanthera longifolia, Cypripedium calceolus, Dactylorhiza incarnata (L.) Soo., Dactylorhiza fuchsii, Epipactis helleborine, Epipactis atrorubens, Neottia nidus-avis, Platanthera bifolia, Platanthera chlorantha, Betula humilis, Genistella sagittalis, Salix myrtilloides, Astrantia major (Andrienko, et al., 2009).

The Red Data Book of Ukraine provides information on the protection of the following species in the study area: Caldesia parnassifolia (the reserve of the national value "Kormin" and the general zoological reserve of the local value "Devil's Swamp"); Succisella inflexa (landscape reserve "Kormin"); Genistella sagittalis (local reserve "Lopatynska Dibrova").

Having analyzed the literature, herbarium data and materials of our own field studies (Glinska et al. 2017, 2018; Shtokalo et al. 2017, 2018), we have compiled a list of rare and endangered species of pine-oak stands. The list was based on the species listed in the "Red Data Book of Ukraine" and the dominant species of the "Green Data Book of Ukraine", in the Appendices of "The Convention on International Trade in Endangered Species of Wild Fauna and Flora", "Convention on the Conservation of European Wildlife and Natural Habitats" and other international rare plant lists, at "List of rare and endangered plant species in the Volyn region" (Table 1).

Table 1. Rare and endangered species of flora in the lists of species in need of protection.

\section{№ $\quad$ Flora species}

Diphasiastrum camplanatum (L.) Holub

Lycopodium annotinum L.

Caldesia parnassifolia (L.)Parl.

Adonis vernalis L.

Allium ursinum L.

Galanthus nivalis L.

Carex umbrosa Host

Gladiolus imbricatus L.

Iris sibirica L.

Lilium martagon $\mathrm{L}$.

Cephalanthera damasonium (Mill.) Druce

Cephalanthera longifolia (L.) Fritsch.

Cephalanthera rubra (L.) Rich.

Cypripedium calceolus $\mathrm{L}$.

Dactylorhiza incarnata (L.) Soo

Dactylorhiza fuchsii (Druce) Soo

\section{旁}

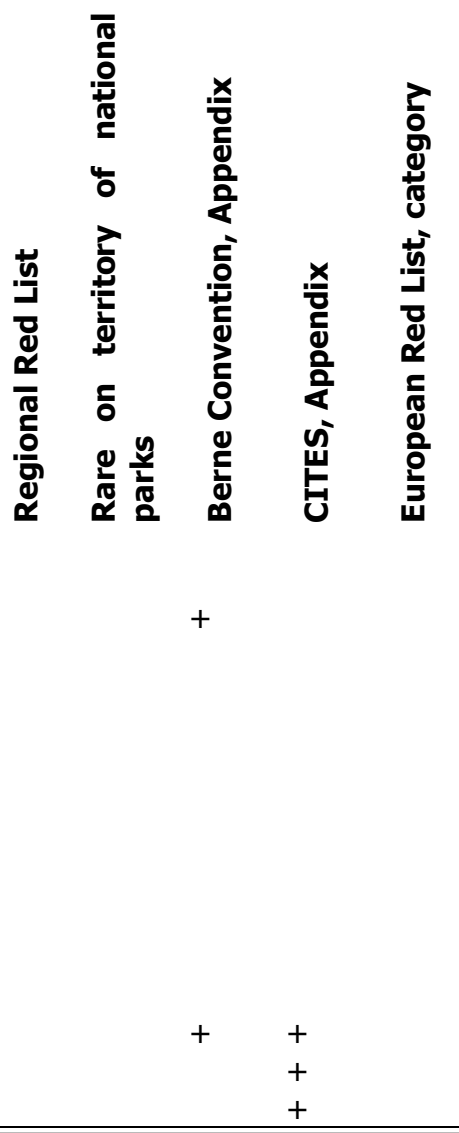


Dactylorhiza majalis (Rich.) P.F. Hunt et Summerhayes

Epipactis palustris (L.) Crantz

Epipactis helleborine (L.) Crantz

Epipactis atrorubens (Hoffm. ex Bernh.) Besser

Neottia nidus-avis (L.) Rich.

Platanthera bifolia (L.) Rich.

Platanthera chlorantha (Cust.) Rchb.

Betula humilis Schrank

Silene lithuanica Zapal.

Succisella inflexa (Kluk) G. Beck

Genistella sagittalis (L.) Gams

Salix myrtilloides L.

Trapa natans $\mathrm{L}$.

Aconitum variegatum $\mathrm{L}$.

Astrantia major L.

Anemone sylvestris L.

Polypodium vulgare $\mathrm{L}$.

Vinca minor L.

Lerchenfeldia flexuosa (L.) Schur

Daphne mezereum $\mathrm{L}$.

Wolffia arrhiza (L.) Horkel ex Wimmer

Ophioglossum vulgatum L.

Actaea spicata L.

Gymnocarpium dryoptheris (L.) Newm.

Campanula cervicaria L.

Campanula sibirica L.

Genista germanica L.

Chimaphylla umbellata (L.) W. Barton

Dentaria glandulosa Waldst. et Kit.

Melittis sarmatica L.

Polygala amarella Crantz

Trifolium alpestre $\mathrm{L}$.

Thfolium montanum L.

Acer pseudoplatanus L.

Cimicifuga europaea Schipcz.

Trollius europaeus $\mathrm{L}$.

Ceratophyllum submersum L.

Digitalis grandiflora Mill.

Aquilegia vulgaris L.

Potentilla alba L.

Hepatica nobilis Mill.

Hedera helix $\mathrm{L}$.

Primula elatior (L.) Hill.

Dentaria bulbifera L.

Nimphaea alba L.

Nimphaea candida C. Presl.

Gypsophilla paniculata L.

Clematis recta $\mathrm{L}$.

Thesium linifolium $\mathrm{L}$.

Pyrethrum corymbosum (L.) Scop.

Eryngium planum L.

Sempervivum ruthenicum Schnittsp. et C.B. Lehm.

Inula helenium $\mathrm{L}$.

Melampyrum arvense $\mathrm{L}$.

Campanula persicifolia L.

Lonicera xylosteuma L.

Isopyrum thalictroides $\mathrm{L}$.

Corydalis cava (L.) Schweigg et Koerte

Spergula morisonii Boreau

Scorzonera humilis L.

Scorzonera purpurea L.

Prunella grandiflora Scholl.

Gentiana cruciata L.

Gentiana pneumonanthe $\mathrm{L}$.

Asparagus officinalis $\mathrm{L}$.

Allium vineale $\mathrm{L}$.

Circaea intermedia Ehrh.

Stachys recta $\mathrm{L}$.

Dryopteris austriaca (Jacq.)Woynar ex Schinz et Thell. 


\begin{tabular}{|c|c|c|c|c|c|c|}
\hline Dianthus pseudosquarrossus (Novak) Klok. & & & + & & & \\
\hline Pulmonaria angustifolia L. & & & + & & & \\
\hline Juniperus communis L. & & & + & & & \\
\hline Total & 29 & 56 & 4 & 3 & 10 & 1 \\
\hline
\end{tabular}

As shown in Table 1, 89 rare species habitats have been found in pine-oak stands, 29 of them are in the Red Data Book of Ukraine, and Silene lithuanica is listed in the European Red List. 3 species (Cypripedium calceolus, Trapa natans ta Caldesia parnassifolia) are included into Appendix of "The Convention on International Trade in Endangered Species of Wild Fauna and Flora". 10 species are subject to protection according to the Appendix of "Convention on the Conservation of European Wildlife and Natural Habitats" 56 species are regionally rare species for the flora of the Volyn region, 4 species are rare species for pine-oak stands. To monitor the status of populations of rare flora species, Botanical Permanent Sampling Areas (BA) have been established in the

Nature conservation Research Branches (NCRB) of Kivertsi National Nature Park "Tsumanska Pushcha":

BA - 1 - Lopatyn NCRB SE "Tsuman Forestry" Partisan Forestry, quarter 11, section 1, with an area of 22 hectares

BA - 2- Lopatyn NCRB Berestyane Forestry, quarter 9, section 24, 3.0 hectares.

BA - 3 - Lopatyn NCRB SE "Tsuman Forestry", quarter 6, section 34, 2.8 hectares.

BA - 4 - Lopatyn NCRB SE "Tsuman Forestry", quarter 2, section 5, 4.1 hectares.

BA - 5 - Lopatyn NCRB SE "Tsuman Forestry", quarter 41, section 6, 1.3 hectares.

BA - 6 - Zviriv NCRB SE "Tsuman Forestry" Moshchanytsia forestry, quarter 74.

BA - 7 - Ozersk NCRB Volyn Forestry of SE "Lviv Military Forest Multi-unit Enterprise", quarter 38, section 15, 3.0 hectares.

BA - 8- Ozersk NCRB Volyn Forestry of SE "Lviv Military Forest Multi-unit Enterprise", quarter 38, section 13, 3.0 hectares.

BA - 9 - Zviriv NCRB SE "Tsuman Forestry" Horyn forestry, quarter 49.

BA - 10 - Lopatyn NCRB Berestyane Forestry, Kormin area, quarter 25, section 6, 5.7 hectares.

BA - 11 - Lopatyn NCRB Berestyane Forestry, Kormin area, quarter 44, section 26.

BA - 12 - Lopatyn NCRB Berestyane Forestry, Kormin area, quarter 25, section 20, 2.2 hectares.

BA - 13 - Lopatyn NCRB SE "Tsuman Forestry" Partisan Forestry, quarter 26, section 3, with an area of 25.5 hectares.

BA - 14 - Lopatyn NCRB SE "Tsuman Forestry" Partisan Forestry, quarter 26, section 3, with an area of 25.5 hectares.

BA - 15 - Ozersk NCRB Volyn Forestry of SE "Lviv Military Forest Multi-unit Enterprise", quarter 38, section 15, with an area of 3.0 hectares.

BA - 17 - Ozersk NCRB SE "Kivertsi Forestry" Trostianets forestry, quarter 58.

Population age range, density, and renewal index (RI) were analyzed for the species studied (Table 2).

Table 2. Age range of populations of rare flora species in permanent sampling areas.

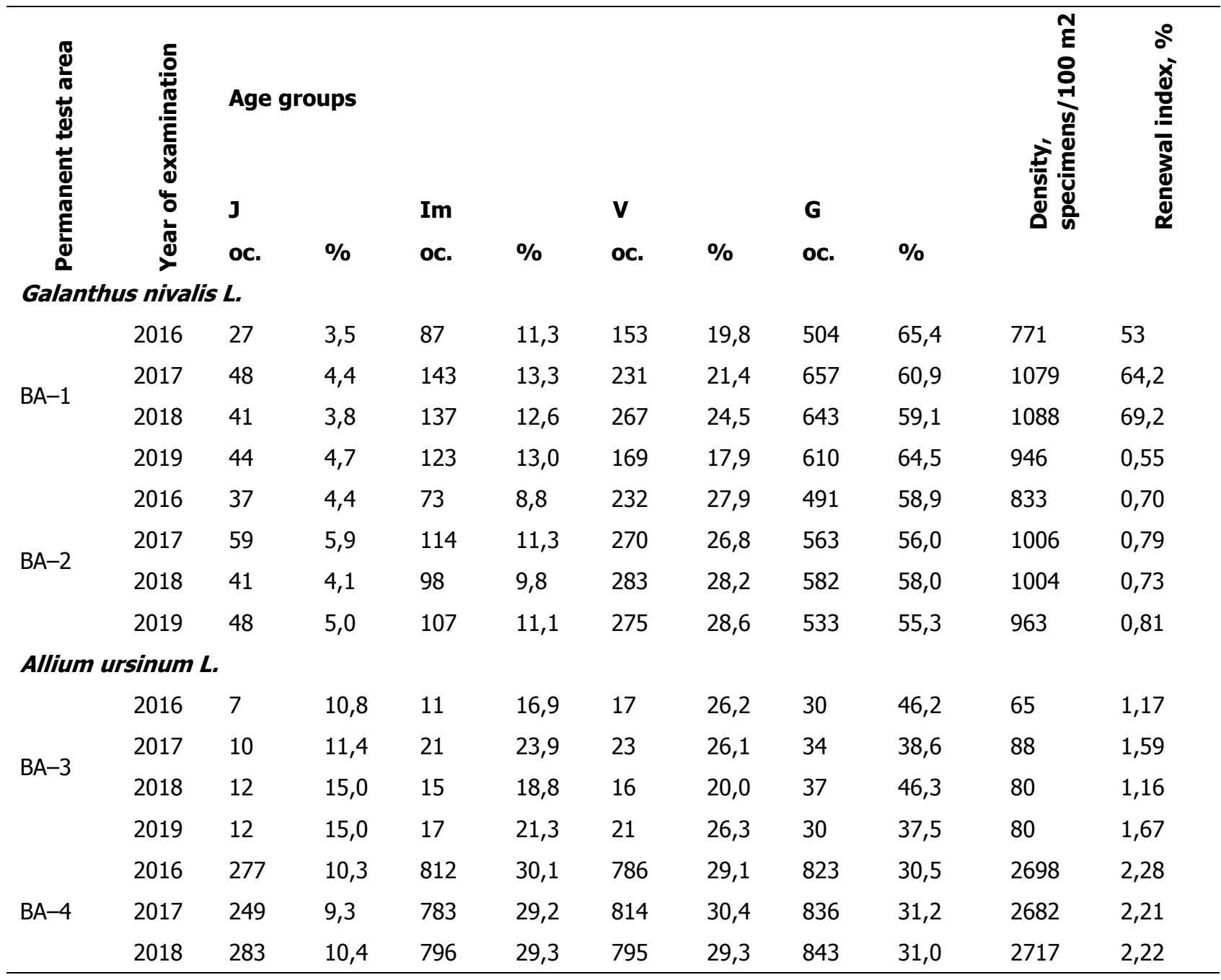




\begin{tabular}{|c|c|c|c|c|c|c|c|c|c|c|c|}
\hline & 2019 & 261 & 9,9 & 754 & 28,5 & 801 & 30,3 & 831 & 31,4 & 2647 & 2,19 \\
\hline \multirow{4}{*}{ BA-5 } & 2016 & 373 & 9,4 & 2536 & 63,7 & 719 & 18,0 & 356 & 8,9 & 3984 & 10,19 \\
\hline & 2017 & 389 & 9,6 & 2448 & 60,4 & 746 & 18,4 & 469 & 11,6 & 4052 & 7,64 \\
\hline & 2018 & 253 & 6,5 & 2359 & 60,9 & 783 & 20,2 & 476 & 12,3 & 3871 & 7,13 \\
\hline & 2019 & 271 & 7,1 & 2275 & 59,7 & 802 & 21,0 & 465 & 12,2 & 3813 & 7,20 \\
\hline \multicolumn{12}{|c|}{ Dentaria glandulosa Waldst. et Kit. } \\
\hline \multirow{4}{*}{$B A-4$} & 2016 & 17 & 2,9 & 21 & 3,6 & 225 & 38,6 & 320 & 54,9 & 583 & 0,82 \\
\hline & 2017 & 21 & 3,5 & 23 & 3,8 & 235 & 38,8 & 327 & 54,0 & 606 & 0,85 \\
\hline & 2018 & 12 & 2,0 & 26 & 4,4 & 253 & 42,9 & 299 & 50,7 & 590 & 0,97 \\
\hline & 2019 & 16 & 2,7 & 29 & 4,8 & 251 & 41,8 & 305 & 50,7 & 601 & 0,97 \\
\hline \multicolumn{12}{|c|}{ Dentaria bulbifera $L$. } \\
\hline \multirow{4}{*}{ BA-3 } & 2016 & 16 & 1,3 & 21 & 1,7 & 759 & 61,9 & 430 & 35,1 & 1226 & 1,85 \\
\hline & 2017 & 21 & 1,6 & 34 & 2,7 & 783 & 61,2 & 441 & 34,5 & 1279 & 1,90 \\
\hline & 2018 & 11 & 0,9 & 42 & 3,3 & 753 & 58,4 & 483 & 37,5 & 1289 & 1,67 \\
\hline & 2019 & 7 & 0,6 & 28 & 2,2 & 742 & 58,5 & 491 & 38,7 & 1268 & 1,58 \\
\hline \multirow{4}{*}{$\mathrm{BA}-4$} & 2016 & 5 & 2,3 & 14 & 6,3 & 56 & 25,3 & 146 & 66,1 & 221 & 0,51 \\
\hline & 2017 & 4 & 1,8 & 9 & 3,9 & 61 & 26,8 & 154 & 67,5 & 228 & 0,48 \\
\hline & 2018 & 9 & 4,1 & 12 & 5,5 & 73 & 33,2 & 126 & 57,3 & 220 & 0,75 \\
\hline & 2019 & 15 & 6,1 & 14 & 5,7 & 54 & 22,1 & 161 & 66,0 & 244 & 0,52 \\
\hline
\end{tabular}

Isopyrum thalictroides $L$.

$\begin{array}{llllllllllll} & 2016 & 2 & 1,3 & 3 & 1,9 & 14 & 9,1 & 135 & 87,7 & 154 & 0,14 \\ \text { BA - 3 } & 2017 & 3 & 1,8 & 6 & 3,6 & 17 & 10,2 & 141 & 84,4 & 167 & 0,18 \\ & 2018 & 6 & 3,4 & 5 & 2,8 & 14 & 7,9 & 153 & 86,0 & 178 & 0,16 \\ & 2019 & 7 & 4,0 & 6 & 3,4 & 18 & 10,2 & 146 & 82,5 & 177 & 0,21 \\ & 2016 & 8 & 3,4 & 11 & 4,7 & 57 & 24,3 & 159 & 67,7 & 235 & 0,48 \\ \text { BA -4 } & 2017 & 3 & 1,3 & 9 & 4,0 & 46 & 20,4 & 167 & 74,2 & 225 & 0,35 \\ & 2018 & 1 & 0,4 & 5 & 2,1 & 59 & 25,0 & 171 & 72,5 & 236 & 0,38 \\ & 2019 & 5 & 2,3 & 7 & 3,3 & 48 & 22,4 & 154 & 72,0 & 214 & 0,39 \\ & 2016 & 8 & 3,3 & 12 & 5,0 & 38 & 15,8 & 182 & 75,8 & 240 & 0,32 \\ \text { BA -5 } & 2017 & 5 & 1,9 & 10 & 3,8 & 47 & 18,1 & 198 & 76,2 & 260 & 0,31 \\ & 2018 & 3 & 1,1 & 7 & 2,5 & 51 & 18,5 & 214 & 77,8 & 275 & 0,29 \\ & 2019 & 4 & 1,5 & 15 & 5,5 & 46 & 17,0 & 206 & 76,0 & 271 & 0,32\end{array}$

Anemone sylvestris $L$.

$\begin{array}{llllllllllll} & 2017 & & 0,0 & 217 & 15,1 & 813 & 56,6 & 407 & 28,3 & 1437 & 2,53 \\ \text { BA-6 } & 2018 & 15 & 0,9 & 243 & 15,1 & 897 & 55,9 & 451 & 28,1 & 1606 & 2,56 \\ & 2019 & 21 & 1,4 & 267 & 18,0 & 805 & 54,2 & 393 & 26,4 & 1486 & 2,78\end{array}$

Cephalanthera damasonium (Mill.) Druce

$\begin{array}{lllllllllll} & 2017 & 0,0 & 1 & 2,4 & 8 & 19,0 & 33 & 78,6 & 42 & 0,27 \\ \text { BA - 7 } & 2018 & 0,0 & 1 & 2,4 & 6 & 14,3 & 35 & 83,3 & 42 & 0,20 \\ & 2019 & 0,0 & & 0,0 & 4 & 22,2 & 14 & 77,8 & 18 & 0,29\end{array}$

Epipactis helleborine (L.) Crantz.

$\begin{array}{lllllllllll} & 2017 & 0,0 & 1 & 12,5 & 2 & 25,0 & 5 & 62,5 & 8 & 0,60 \\ \text { BA-7 } & 2018 & 0,0 & & 0,0 & 1 & 12,5 & 7 & 87,5 & 8 & 0,14 \\ & 2019 & 0,0 & & 0,0 & 2 & 28,6 & 5 & 71,4 & 7 & 0,40 \\ & 2017 & 0,0 & 1 & 3,1 & 4 & 12,5 & 27 & 84,4 & 32 & 0,19 \\ \text { "At } & 2018 & 0,0 & & 0,0 & 2 & 7,4 & 25 & 92,6 & 27 & 0,08 \\ \text { Panas's" } & 2019 & 0,0 & & 0,0 & 1 & 3,3 & 29 & 96,7 & 30 & 0,03 \\ & 2019\end{array}$




\section{Lilium martagon L.}

$\begin{array}{lllllllllll} & 2017 & 0,0 & 8 & 8,4 & 51 & 53,7 & 36 & 37,9 & 95 & 1,64 \\ \text { BA }-8 & 2018 & 0,0 & 3 & 3,1 & 57 & 58,8 & 37 & 38,1 & 97 & 1,62 \\ & 2019 & 0,0 & & 0,0 & 9 & 90,0 & 1 & 10,0 & 10 & 9,00\end{array}$

Dactilorhiza majalis (Rchb.) Hult. et Summerhayer

$\begin{array}{lllllllllll}\text { BA - 9 } & 2018 & 0,0 & 5 & 11,1 & 12 & 26,7 & 28 & 62,2 & 45 & 0,61 \\ & 2019 & 0,0 & 1 & 3,6 & 11 & 39,3 & 16 & 57,1 & 28 & 0,75 \\ \text { BA - 10 } & 2018 & 0,0 & 2 & 14,3 & 5 & 35,7 & 7 & 50,0 & 14 & 1,00 \\ & 2019 & 0,0 & & 0,0 & 6 & 60,0 & 4 & 40,0 & 10 & 1,50 \\ \text { BA - 11 } & 2018 & 0,0 & 1 & 8,3 & 3 & 25,0 & 8 & 66,7 & 12 & 0,50 \\ & 2019 & 0,0 & & 0,0 & 5 & 50,0 & 5 & 50,0 & 10 & 1,00\end{array}$

\section{Dactilorhiza incarnata (L.) Soo'}

$\begin{array}{llllllllllll}\mathrm{BA}-11 & 2018 & 0,0 & 1 & 12,5 & 2 & 25,0 & 5 & 62,5 & 8 & 0,60 \\ & 2019 & 0,0 & & 0,0 & 1 & 16,7 & 5 & 83,3 & 6 & 0,20\end{array}$

Iris sibirica $\mathrm{L}$.

$\begin{array}{llllllllll}\text { BA - 12 } & 2018 & 0,0 & 0,0 & 1 & 20,0 & 4 & 80,0 & 5 & 0,25 \\ & 2019 & 0,0 & 0,0 & 2 & 25,0 & 6 & 75,0 & 8 & 0,33\end{array}$

Betula obscura A. Kotula

$\begin{array}{llllllllll}\text { BA }-13 & 2018 & 0,0 & 0,0 & 0,0 & 4 & 100,0 & 4 & 0,00 \\ & 2019 & 0,0 & 0,0 & 0,0 & 1 & 100,0 & 1 & 0,00\end{array}$

Platanthera bifolia (L.) Rich.

$\begin{array}{lllllllllll}\text { BA - 14 } & 2018 & 0,0 & 3 & 11,5 & 7 & 26,9 & 16 & 61,5 & 26 & 0,63 \\ & 2019 & 0,0 & 5 & 14,3 & 12 & 34,3 & 18 & 51,4 & 35 & 0,94\end{array}$

Astrantia major L.

\begin{tabular}{|c|c|c|c|c|c|c|c|c|c|c|c|}
\hline \multirow{4}{*}{$B A-15$} & 2016 & 5 & 7,8 & 8 & 12,5 & 34 & 53,1 & 17 & 26,6 & 64 & 2,76 \\
\hline & 2017 & 2 & 3,1 & 4 & 6,3 & 39 & 60,9 & 19 & 29,7 & 64 & 2,37 \\
\hline & 2018 & 6 & 8,1 & 10 & 13,5 & 32 & 43,2 & 26 & 35,1 & 74 & 1,85 \\
\hline & 2019 & 4 & 5,6 & 7 & 9,7 & 38 & 52,8 & 23 & 31,9 & 72 & 2,13 \\
\hline \multicolumn{12}{|c|}{ Silene Iithuanica Zapal. } \\
\hline \multirow{2}{*}{$B A-17$} & 2018 & 1 & 3,3 & 4 & 13,3 & 4 & 13,3 & 21 & 70,0 & 30 & 0,43 \\
\hline & 2019 & 3 & 7,0 & 5 & 11,6 & 6 & 14,0 & 29 & 67,4 & 43 & 0,48 \\
\hline
\end{tabular}

Studies have found that the highest population density recorded was the following: Allium ursinum - 4052 specimens/100 $\mathrm{m}^{2}$; Anemone sylvestris - 1606 specimens $/ 100 \mathrm{~m}^{2}$; Dentaria bulbifera - 1289 specimens/100 m²; Galanthus nivalis - 1088 specimens $/ 100 \mathrm{~m}^{2}$; Dentaria glandulosa - 606 specimens $/ 100 \mathrm{~m}^{2}$. Populations of Orchidaceae have low quantity and density, rightsided spectra of ontogenetic states dominated by generative individuals: Cephalanthera damasonium - 18-42 specimens/100 $\mathrm{m}^{2}$; Epipactis helleborine - 7-32 specimens/100 $\mathrm{m}^{2}$; Dactilorhiza majalis - 10-45 specimens/100 $\mathrm{m}^{2}$; Dactilorhiza incarnata - 6-8 specimens $/ 100 \mathrm{~m}^{2}$; Platanthera bifolia - 26-35 specimens/100 $\mathrm{m}^{2}$. Population Betula obscura consist of 1 tree about 80 years old, seed reproduction is not observed. In January 2019, three trees were killed as a result of the storm.

\section{Conclusion}

Having analyzed the literary information, herbarium data and materials of our own field research, we have compiled a list of rare and endangered species of pine-oak tree stands of 89 species. The study found that for 63 rare species population dynamics and distribution of species is satisfactory. The area of distribution and the quantity of 7 species is increasing: Allium ursinum, Galanthus nivalis, Platanthera chlorantha, Anemone sylvestris, Campanula persicifolia, Corydalis cava, Isopyrum thalictroides. In the study area 14 species grow sporadically: Juniperus communis, Potentilla alba, Digitalis grandiflora, Gymnocarpium dryoptheris, Daphne mezereum, Neottia nidus-avis, Epipactis helleborine, Scorzonera purpurea, Asparagus officinalis, Iris sibirica, Adonis vernalis, Cephalanthera damasonium, Gentiana cruciate, Gentiana pneumonanthe. Distribution information on Caldesia parnassifolia, Succisella inflexa, Genistella sagittalis, Salix myrtilloides, Ophioglossum vulgatum is insufficient to establish the species dynamics and needs further investigation. The importance of preserving the species in this area is difficult to quantify. It is extremely important to preserve species that are confined to meadows and wetlands specific to the study area: Gladiolus imbricatus, Iris sibirica, Dactylorhiza incarnata, Dactylorhiza fuchsii, Dactylorhiza majalis, Epipactis palustris, Betula humilis, Succisella inflexa, Genistella sagittalis, Salix myrtilloides, Trapa natans. Preservation of pine-oak stands of Volyn Polissya will help to create conditions for growth: Diphasiastrum camplanatum, Cephalanthera damasonium, Cephalanthera longifolia, Cephalanthera rubra, Epipactis 
helleborine, Epipactis atrorubens, Neottia nidus-avis, Platanthera bifolia, Platanthera chlorantha, Silene lithuanica, Dianthus pseudosquarrossus, Cimicifuga europaea, Primula elatior

\section{References}

Andriienko, T. L., Konishchuk, V. V. \& Priadko, O. I. (2009). Ridkisni vydy sudynnykh roslyn Volynskoi oblasti [Rare species of vascular plants of Volyn Region]. Zapovidna sprava v Ukraini [Nature Conservation in Ukraine]. 15(2), 20-26. [in Ukrainian].

Andriienko, T. L., Kliestov, M. L., Khymyn, M. V., Priadko, O. I., Onyshchenko, V. A., Kot, A.S. \& Hryhorenko, S.O. (2004). Bioriznomanittia Tsumanskoi pushchi ta pytannia yoho zberezhennia [The biodiversity of Tsumanska Pushcha and its conservation issues]. Kyiv: Fitosotsiolohichnyi tsentr [in Ukrainian].

Glinska, S., Shvets, H., Shtokalo, S. \& Nykytiuk, T. (2017). Rarytetni vydy flory Kivertsivskoho natsionalnoho pryrodnoho parku "Tsumanska pushcha" [Rare species of flora of Kivertsi National Nature Park "Tsumanska Pushcha"]. Mynule i suchasne Volyni ta Polissia. Kivertsivshchyna ta Olyka v istorii Ukrainy ta Volyni [Past and present of Volhynia and Polissia. Kivertsi district and Olyka in the history of Ukraine and Volyn]. 62, 439 - 443. [in Ukrainian].

Glinska, S.O, Shvets, H.I. \& Shtokalo, S.S. (2018). Poshyrennia rehionalno-ridkisnykh vydiv u Volynskii oblasti [The distribution of regionally rare species in Volyn region] The 2d International Scientific and Practical Conference "Problemy ekolohii ta evoliutsii ekosystem v umovakh transformovanoho seredovyshcha" [Problems of Ecology and Evolution of Ecosystems in Transformed Environment] (11-12.10.2018, Chernihiv). Chernihiv : Desna Polihraf. 185-188. [in Ukrainian].

The Convention on International Trade in Endangered Species of Wild Fauna and Flora (1999). Kyiv. [in Ukrainian].

Convention on the Conservation of European Wildlife and Natural Habitats (1998). Kyiv. [in Ukrainian].

Pachoskii, I.K. (1897; 1899; 1900) Flora Polesia i prilezhashchikh mestnostei [Flora Polesia and surrounding areas]. Trudy SanktPeterburgskogo obshchestva estestvoispytatelei [Transactions of St. Petersburg Naturalist Society]. 27 (2); 29 (3); 30 (3). [in Russian].

Rabotnov, T. A. (1978). Fitotcenologiia [Phytocoenology]. - Moscow: Izdatelstvo MGU [in Russian].

Spysok rehionalno ridkisnykh, znykaiuchykh vydiv roslyn, hrybiv i tvaryn, yaki potrebuiut okhorony u Volynskii oblasti [The list of regionally rare, endangered species of plants, fungi and animals in need of protection in Volyn region] Retrieved from http://volynrada.gov.ua/session/29/30

Uranov, A. A. (1975). Vozrastnoi spektr fitotcenopopuliatcii kak funktciia vremeni i energeticheskikh volnovykh protcessov [The age spectrum of the phytocenopopulation as a function of time and energy wave processes]. Biologicheskie nauki [Biological Sciences]. 2, 7 - 34. [in Russian].

Didukh, Ya. P. (Ed.). (2009). Chervona knyha Ukrainy. Roslynnyi svit [The Red Book of Ukraine. Flora]. K. : Hlobalkonsaltynh [in Ukrainian].

Shtokalo, S. S., Glinska, S. O., Rozman, M. V., Ohorodnyk, O. V. \& Shvets, H. I. (2018). Monitorynh rarytetnykh vydiv flory Kivertsivskoho natsionalnoho pryrodnoho parku "Tsumanska pushcha" [Monitoring of rare flora species of Kivertsi National Natural Park "Tsumanska Pushcha"]. International Scientific and Practical Conference "Pryrodookhoronni, ekoosvitni, rekreatsiino-turystychni ta istoryko-kulturni aspekty staoloho rozvytku Roztochchia" [Environmental, eco-educational, recreational, tourist and historicalcultural aspects of sustainable development of Roztoche] (4.07.2018, Ivano-Frankove). 209 - 212. [in Ukrainian].

Shtokalo, S. S., Ralo, V. M. \& Glinska S. O. (2017). Ridkisni ta znykaiuchi vydy pryrodnoi flory Kivertsivskoho natsionalnoho pryrodnoho parku "Tsumanska pushcha" [Rare and endangered species of the natural flora of Kivertsi National Nature Park "Tsumanska Pushcha"]. Scientific and Practical Conference with International Participation "Voda: Problemy ta shliakhy vyrishennia" [Water: Problems and Solutions]. (05-08.07. 2017, Rivne). Zhytomyr: Vyd-vo ETs "Ukrekobiokon". [in Ukrainian].

Macko, S. (1937). Roślinność projektowanych rezerwatów na Wołyniu. Ochrona Pryrody. 18, 111-185. [in Polish].

Vandas, K. (1886). Ein Beitrag zur Kenntnis der Flora Wolhynien. Öester. Bot. Z. 34, 34, 155-157, 192 - 195. [in German].

\section{Citation:}

Glinska, S.O., Shtokalo, S.S., Lyko, D.V., Stepaniuk, Ya.V., Savchuk, L.K. (2020). Ecological-coenotic features of rare flora species of pine-oak stands of Volyn Polissya. Under urban ecosystem. Ukrainian Journal of Ecology, 10(1), 55-61.

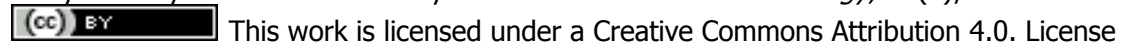

